\title{
Digital multicarrier modulation techniques for high data rates multimedia applications in present and future wireless communication systems
}

\author{
Rabindra Bhojray ${ }^{1}$, Sumant kumar Mohapatra ${ }^{2}$ \\ Department of Electronics and Telecommunication Engg. Trident Academy of Technology BPUT, \\ Bhubaneswar, Odisha, India
}

\begin{abstract}
To accommodate high performance and high bit rates for multimedia applications in present and future wireless communication systems, it is essential that modulation techniques applied in this scenario are able to support high data rates upto and above $200 \mathrm{Mbps}$. To meet the increased demand of higher bit rates, the wireless systems are incorporating the multi-carrier modulation techniques, such as FFT based MCM which is also known as conventional OFDM and wavelet transform based MCM. Wireless multicarrier modulation is a technique of transmitting data by dividing the input data stream into parallel sub-streams that are each modulated and multiplexed onto the channel at different carrier frequencies. In this paper, we studied the performance of DWT based MC-CDMA and FFT based MC-CDMA modulations for transmission over wireless fading channels. This scheme is shown to be overall quite similar to FFT based OFDM modulation, but with some interesting additional features and improved characteristics. Results shows that DWT based MCCDMA modulation has better bit error rate (BER) performance than conventional FFT based CDMA without cyclic prefix (CP) for all signal-to-noise ratios and also outperforms FFT based CDMA for low signal-to-noise ratio values.
\end{abstract}

Key words: Code division multiple accesses, discrete wavelet transform, Multicarrier modulation, Orthogonal Frequency Division Multiplexing,

\section{Introduction}

In the current and future mobile communications systems, data transmission at high bit rates is essential for many services such as high quality audio and video and mobile integrated service digital network [1]. For systems beyond 3G, there may be a requirement for a new wireless access technology for the terrestrial components. It's envisaged that these potential new radio interfaces will support up to approximately100 Mbps for high mobility and up to $1 \mathrm{Gbps}$ for the low mobility users. The principle of multicarrier modulation is not recent, its actual use in commercial systems had been delayed until the technology required to implement it became available at reasonable cost [2]. In multicarrier modulation, the essential requirement on the elementary pulse shaping is the orthogonality with its time-frequency shifted versions. However, the propagation over the wireless channel leads to the loss of orthogonality, which could produce intersymbol (ISI) and interchannel interferences (ICI). In a multipath environment with time dispersion, it has been proven that multicarrier transmission using OFDM modulation is very efficient [3]. Similarly, the idea of using more advanced transform than Fourier's as the core of a multicarrier system has been introduced more than a decade ago [4], [5]. However, such alternative methods have not been foreseen as of major interest and therefore have received little attention. With the current demand for high performance in wireless communication systems, we are entitled to wonder about the possible improvement that Discrete wavelet Transform-based modulation could exhibit compared to Discrete Fourier transform based modulation [6].

The Orthogonal Frequency Division Multiplexing (OFDM) appeared in the 1990s and gained a lot of attention, it provides an efficient means to handle high speed data streams over a multipath fading environment and is a potential candidate for $4 \mathrm{G}$ systems. OFDM is very efficient in spectrum usage and is very effective in a frequency selective channel[7],[8]. A variation of OFDM which allows multiple accesses is MultiCarrier CDMA (MC-CDMA) which is essentially an OFDM technique where the individual data symbols are spread using spreading code in frequency domain. The inherent processing gain due to the spreading helps in interference suppression in addition to providing high data rates. Multicarrier techniques can combat hostile frequency selective fading countered in mobile communications. The robustness against frequency selective fading is very attractive, especially for high-speed data transmission. Enhancement of robustness against frequency selective fading and high scalability in possible data transmission rate is achieved by combining OFDM with CDMA [9],[10]. OFDM can provide higher data rates as is a very good choice for service providers to compete with wire-line carriers [11]. Combining multi-carrier OFDM transmissions with Code Division 
Multiple Accesses (CDMA) allows us to exploit the wideband channels inherent frequency diversity by spreading each symbol across multiple carriers.

Although OFDM is robust to frequency selective fading, it has severe disadvantages in subcarrier synchronization and sensitivity to frequency offset estimation. The other one is related with the presence of a large number of sub-carriers which exhibit a non-constant nature in its envelope. The combining of OFDM and CDMA has one major advantage since; it can lower the symbol rate in each sub-carrier compared to OFDM so that longer symbol duration makes it easier to synchronize. The MC-CDMA not only mitigates the Inter-Symbol Interference (ISI) but also exploits the multipath. The MC-CDMA suffers only slightly in presence of interference as opposed to Direct Sequence-CDMA (DS-CDMA) whose performance decreases significantly in the presence of interference [12],[13] . Wavelet theory has been foreseen by several authors as a good platform on which to build multicarrier waveform bases [4], [14]. Wavelet packet bases therefore appear to be a more logical choice for building orthogonal waveform sets usable in communication,. In wavelet based OFDM, CP is not required ,CP is $20 \%$ or more of symbol , thus wavelet based OFDM gives $20 \%$ or more bandwidth efficiency [15]. Wavelet based OFDM is less affected by Doppler shift. In wavelet based OFDM a prototype wavelet filter provides both orthogonality and good time-frequency localization. Wavelet provides phase linearity and significant out-of-band rejection. Its energy compaction is also high. In this paper, we compared the bit error rate (BER)of DWT based MC-CDMA and FFT based MC CDMA modulations in AWGN and Flat fading wireless channels. And also we have evaluated the BER performance of of FFT based MC-CDMA system and DWT based MC-CDMA system with Haar and Daubechies wavelets.

\section{Wavelet Transform}

Wavelet means small wave. Wavelet analysis is analyzing signal with short duration finite energy function. Wavelet transform the signal under analysis into another representation that present the signal in more useful form [5], [26]. It is a tool for studying signals in the joint time-frequency domains. Wavelets have compact support (localization) both in time and frequency domain, and possess better orthogonality . Orthogonal wavelets are capable of reducing the power of inter symbol interference (ISI) and inter carrier interference (ICI) which are caused by loss of orthogonality between the carriers [ 16 ] as a result of multipath propagation over the wireless fading channels.

Equivalent mathematical conditions for wavelet are :

$\int_{-\infty}^{\infty} \Psi(t) d t=0$

This means that the function integrates to zero and is a oscillatory function and there is no need to use cosine and sine waves.

$\int_{-\infty}^{\infty}|\Psi(t)|^{2} d t<\infty$

This means that the function is square integrable or has finite energy. And the admissibility condition is given by

$\int_{-\infty}^{\infty} \frac{|\Psi(\omega)|^{2}}{|\omega|} d \omega<\infty$

where $\Psi(\omega)$ is the Fourier Transform of $\Psi(t)$.A real or continuous function $\psi(t)$ is called a basic wavelet or mother wavelet.

The continuous wavelet transform is defined as:

$\Psi_{a, b}(t)=\frac{1}{\sqrt{a}} \Psi\left(\frac{t-b}{a}\right)$

The parameter a is the scaling parameter or scale, and it measures the degree of compression. The parameter $b$ is the translation parameter which determines the time location of the wavelet. If $|a|<1$, then the wavelet in (4) is the compressed version of the mother wavelet and corresponds mainly to higher frequencies. On the other hand, when $|a|>1$, then $\Psi_{a, b}(t)$ has a larger time-width than $\Psi(t)$ and corresponds to lower frequencies. Thus, wavelets have time-widths adapted to their frequencies. This is the key reason for the success of the wavelets in time-frequency signal analysis and signal processing. 


\section{The Fast Discrete Wavelet Transform}

The fast wavelet transform is a computational efficient implementation of the discrete wavelet transform that exploits a surprising relationship between the coefficients of DWT at adjacent scales [14], [17]. Any function $\mathrm{f}(\mathrm{x})$ could be written as a series expansion in terms of the scaling function and wavelets by:

$$
f(x)=\sum_{k} c_{j_{0}}(k) \varphi_{j_{0}, k}(x)+\sum_{j=j_{0}}^{\infty} \sum_{k} d_{j}(k) \psi_{j, k}(x)
$$

Where $j_{0}$ is an arbitrary starting scale and $c_{j_{0}}(k)$ are known as approximations or scaling coefficients and $d_{j}(k)$ are known as detail or wavelet coefficients. The scaling and wavelet coefficients at scale $\mathrm{j}$ are related to the scaling coefficients at scale $(j+1)$ by the following two relations .

$$
\begin{aligned}
& c_{j}(k)=\sum_{m} h(m-2 k) c_{j+1}(m) \\
& d_{j}(k)=\sum_{m} g(m-2 k) d_{j+1}(m)
\end{aligned}
$$

The implementation of equations (6) and (7) is illustrated in "Fig. 1". In this figure, two levels of decomposition are depicted. $\mathrm{h}$ and $\mathrm{g}$ are low-pass and high-pass filters corresponding to the coefficients $h(n)$ and $g(n)$ respectively. The down-pointing arrows denote a decimation or down-sampling by two. This splitting, filtering and decimation can be repeated on the scaling coefficients to give the two-scale structure [18]. The first stage of two banks divides the spectrum of $c_{j-1, k}$, into a low-pass and high-pass band, resulting in the scaling coefficients and wavelet coefficients at lower scale $\left(c_{j, k}\right)$ and $\left(d_{j, k}\right)$. The second stage then divides that lowpass band into another lower low-pass band and a high-pass filter.

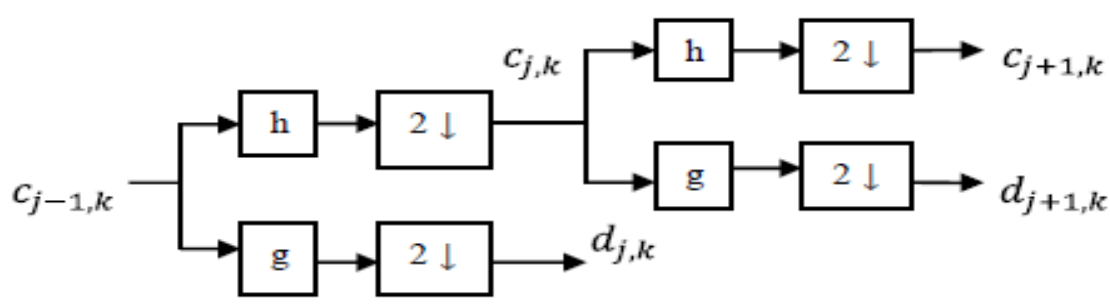

Fig.1. The two stage FWT analysis filter bank

\section{Orthogonal Frequency Division Multiplexing (OFDM)}

OFDM is a combination of multiplexing and modulation. OFDM uses three transmission principles, multirate, multisymbol, and multicarrier . OFDM is identical to coded OFDM (COFDM)), is a frequencydivision multiplexing (FDM) scheme used as a digital multi-carrier modulation method. The main concept in OFDM is orthogonality of the sub-carriers.the orthogonality allows simultaneous trans mission on a lot of sub carriers in a tight frequency space without interference from each other[ 3 ]. One of the major advantages of OFDM is that the modulation can be performed in the discrete domain using an Inverse Discrete Fourier Transform (IDFT) or more computationally efficient inverse Fast Fourier Transform (IFFT). By using a IDFT or IFFT the whole bandwidth will be split into $N$ subchannels [19] . As a result, a high data stream will be transformed into $\mathrm{N}$ - low rate streams, which are transmitted over different sub-channels. OFDM can mitigate the ISI in a frequency-selective channel. Input data stream is QAM or PSK modulated, and divided into blocks of data. Spectrally efficient, overlapped and orthogonal subcarriers carry multiple streams of data symbols in parallel. The parallel transmission of data over multiple simultaneous carriers makes the OFDM system to be more robust against frequency selective fading or narrowband interference [20], some subcarriers may be degraded, others will be unaffected.

The OFDM system has some key advantages: Makes efficient use of the spectrum by allowing overlap, eliminates ISI and ICI through use of a cyclic prefix, pulse shaping could reduce both ICI,ISI and narrow band interference, is less sensitive to sample timing offsets than single carrier systems [8].And drawbacks as OFDM signal has a noise like amplitude with a very large dynamic range and requires RF power amplifiers with a high peak to average power ratio, The FFT based OFDM system is shown in "Fig. 2". The input binary information is first converted into parallel data sequences and each serial/parallel converter output is multiplied with spreading code. Data from all subcarriers is modulated in baseband by inverse fast Fourier transform (IFFT) and converted back into serial data. The guard interval (e.g cyclic prefix ,zero padding etc) is 
inserted between symbols to avoid ISI caused by multipath fading and finally the signal is transmitted after RF up-conversion. At the receiver, after down- conversion, the m-subcarrier component corresponding to the received data is first coherently detected with FFT and then multiplied with gain to combine the energy of the received signal scattered in the frequency domain. Table 1 indicates the data rates and modulation techniques of 802.11 Network Standards.

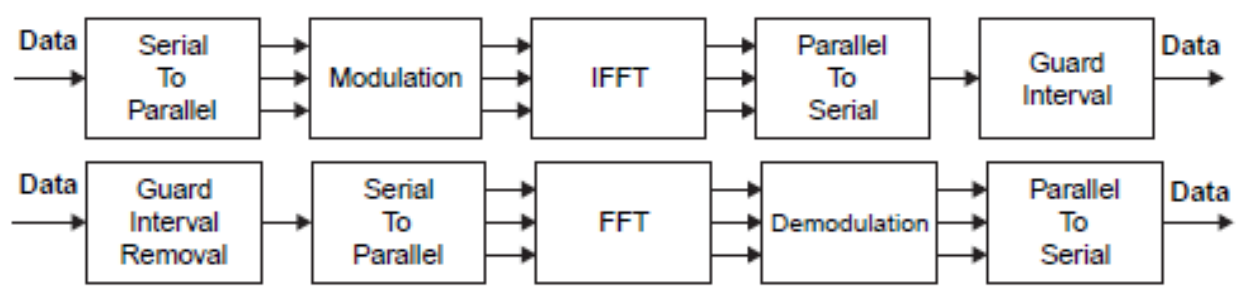

Fig. 2. FFT based OFDM system

Table.1

Summarizes the data rates and modulation techniques of 802.11 Network Standards

\begin{tabular}{|l|l|c|c|c|c|c|}
\hline Protocol & $\begin{array}{c}\text { Year of } \\
\text { Release }\end{array}$ & $\begin{array}{c}\text { Frequency } \\
(\mathrm{GHz})\end{array}$ & $\begin{array}{l}\text { B.W } \\
(\mathrm{MHz})\end{array}$ & $\begin{array}{c}\text { Max data } \\
\text { Rates } \\
(\mathrm{Mbps})\end{array}$ & MIMO & Modulation \\
\hline $802.11 \mathrm{a}$ & Sep 1999 & $5 / 3.7$ & 20 & 54 & 1 & OFDM \\
\hline $802.11 \mathrm{~b}$ & Sep 1999 & 2.4 & 20 & 11 & 1 & DSSS \\
\hline $802.11 \mathrm{~g}$ & Jun 2003 & 2.4 & 20 & 54 & OFDM \\
\hline $802.11 \mathrm{n}$ & Oct 2009 & $2.4 / 5$ & $20 / 40$ & $72 / 150$ & 4 & OFDM \\
\hline $802.11 \mathrm{ac}$ & Dec 2012 & 5 & $80 / 160$ & $433 / 866$ & 8 & OFDM \\
\hline
\end{tabular}

\section{Multi Carrier CDMA Modulation}

Code Division Multiple Access (CDMA) is a multiple access technique, which is based on Spread Spectrum modulation scheme. CDMA allows multiple users to access the channel simultaneously, with each user's data being multiplied by a unique and orthogonal pseudo-random code sequence [9],. In spread spectrum system, the transmitted signal is spread over a wide frequency band, much wider, than the bandwidth of the information being sent. This is accomplished by modulating the information with a wideband encoding signal There are two main ways of spreading a signal [ 10], spreading in time which is usually referred to as Direct Sequence Spread Spectrum (DSSS), and spreading in frequency, also known as Frequency Hopping Spread Spectrum (FHSS).

CDMA and multi-carrier modulation, such as OFDM that can achieve high spectral efficiencybecause the spectrum of successive sub-carriers is allowed to overlap. Although OFDM is robust to frequency selective fading, it has severe disadvantages in subcarrier synchronization and sensitivity to frequency offset estimation. The other one is related with the presence of a large number of sub-carriers which exhibit a non-constant nature in its envelope [21]. But OFDM can provide higher data rates as is a very good choice for service providers to compete with wire-line carriers [11]. The CDMA scheme is robust to frequency selective fading and has been successfully introduced in commercial cellular mobile communications systems such as Interim Standard-95 (IS-95) and 3G systems. Combining multi-carrier OFDM transmissions with Code Division Multiple Accesses (CDMA) allows us to exploit the wideband channels inherent frequency diversity by spreading each symbol across multiple carriers [22],[23]. Also the combining of OFDM and CDMA has one major advantage that it can lower the symbol rate in each sub-carrier compared to OFDM so that longer symbol duration makes it easier to synchronize [12].

The combined CDMA systems with OFDM are mainly categorized into multi tone CDMA(MTCDMA) and multi carrier CDMA(MC-CDMA].In the MT-CDMA scheme, spreading occurs in the time Domain. The incoming data stream is first converted to a parallel stream and then spread in time using spreading codes. This ensures that the resulting spectrum has orthogonal sub-carriers [24].In the MC-CDMA scheme, spreading occurs in the frequency domain. Here multiple copies of the same data symbol each multiplied by one chip of a user specific

spreading code are transmitted on different sub-carriers in parallel over overlapping carriers in the frequency domain[25]. 
The model of DWT based MC-CDMA system is shown in "Fig. 3". The only difference is in conventional FFT based MC-CDMA, we use IFFT/FFT, but in wavelet based MC-CDMA we have to use IDWT instead of IFFT and DWT instead of FFT. Also there is no need of cyclic prefix in wavelet based OFDM.

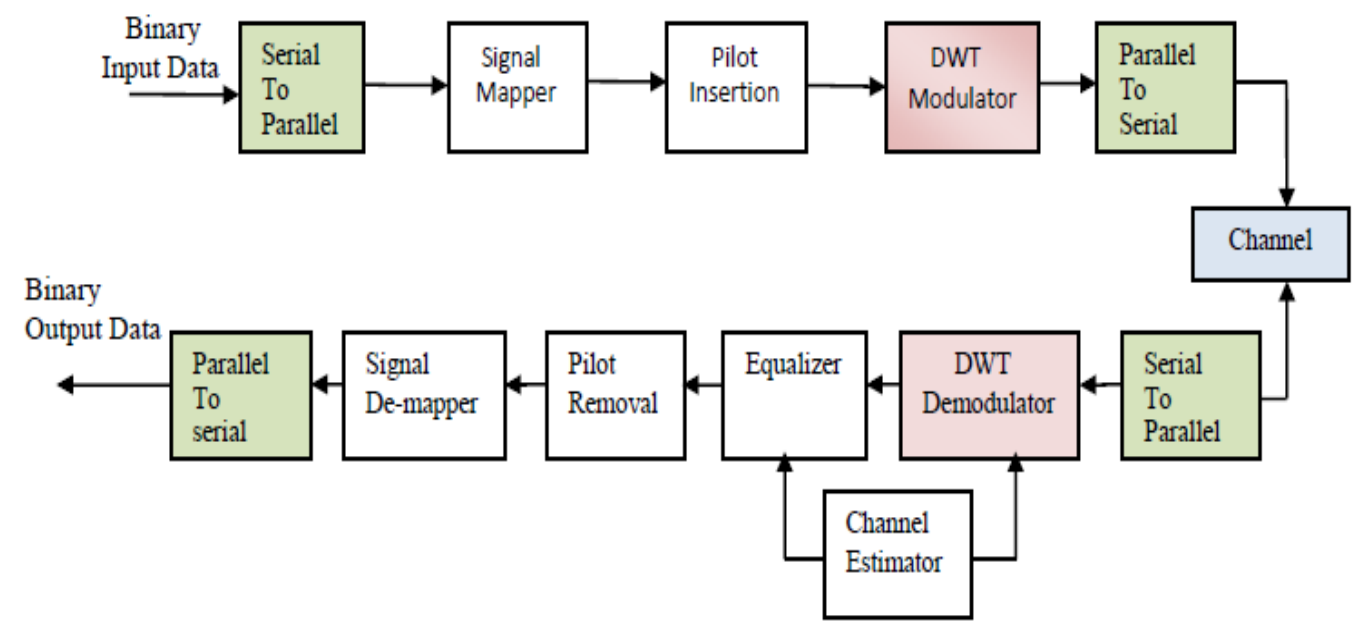

Fig. 3. DWT based MC- CDMA system

\section{Performance Evaluation And Simulation Results}

Computer simulation of the FFT based MC-CDMA system and DWT based MC-CDMA system were carried out to evaluate the performances of the systems using MATLAB. At first we have compared the BER performance of FFT based MC-CDMA system and DWT based MC-CDMA system over both AWGN and flat fading channel. "Figs. 4" and "Fig. 5" show the BER performance of FFT based MC-CDMA and DWT based MC-CDMA system under AWGN channel and flat fading channel respectively. We have taken total of 64 subcarriers in the simulation the band width used for the was $10 \mathrm{MHz}$., Doppler frequency shift is $20 \mathrm{~Hz}$ and the Sub carrier modulation is QPSK. "Fig. 4" shows the BER performance of FFT based MC-CDMA system and DWT based MC-CDMA system with AWGN. From the figure, the DWT based MC-CDMA system has $\mathrm{BER}=10^{-1}$ at SNR approximately $=2.5 \mathrm{~dB}$, while FFT based MC-CDMA system, the has $\mathrm{BER}=10^{-1}$ at approximately $\mathrm{SNR}=19 \mathrm{~dB}$, which means a gain of about $16.5 \mathrm{~dB}$ has been achieved.

"Fig. 5 "shows the BER performance of FFT based MC-CDMA system and DWT based MCCDMA system over and flat fading channel, from the fig. 5 , the DWT based MC-CDMA system has $\mathrm{BER}=10^{-1}$ at approximately $\mathrm{SNR}=5 \mathrm{~dB}$, while FFT based MC-CDMA system, the has $\mathrm{BER}=10^{-1}$ at approximately $\mathrm{SNR}=22.5 \mathrm{~dB}$, which means a gain of about $17.5 \mathrm{~dB}$ has been achieved. Again the DWT based MC-CDMA system has $\mathrm{BER}=10^{-2}$ at approximately $\mathrm{SNR}=15 \mathrm{~dB}$, while FFT based MC-CDMA system, the has $\mathrm{BER}=10^{-2}$ at approximately $\mathrm{SNR}=32 \mathrm{~dB}$, which means a gain of about $17 \mathrm{~dB}$ has been achieved. It is clear from shown figures that DWT based MC-CDMA system better BER performance as compared to FFT based MCCDMA system.

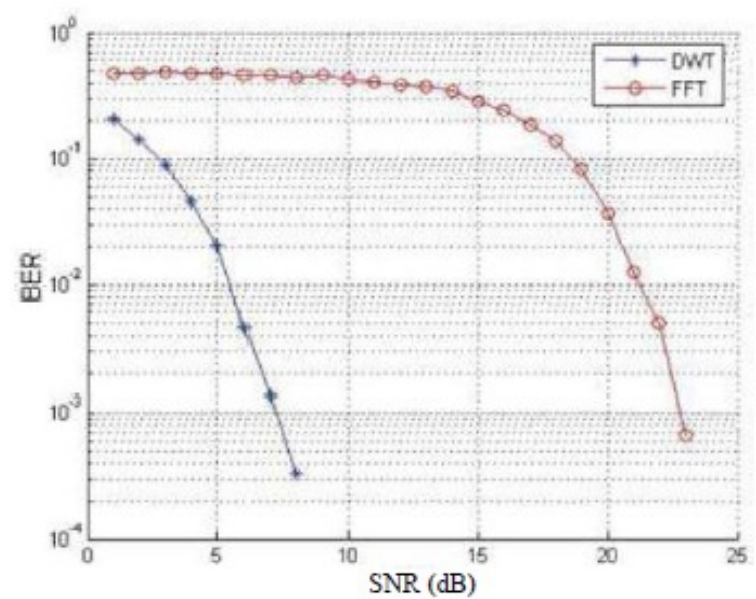

Fig. 4. BER performance of DWT based MC-CDMA system in AWGN channel

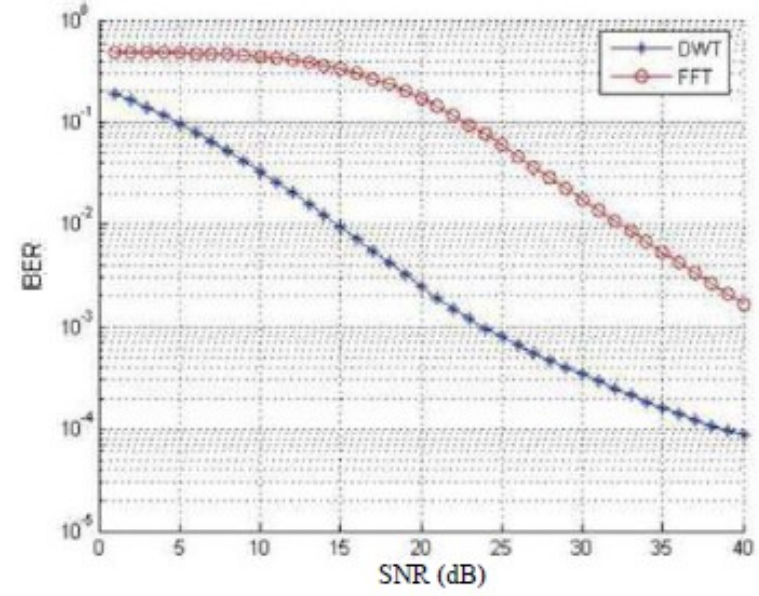

Fig. 5. BER performance of DWT based MC- CDMA system in flat fading channel 


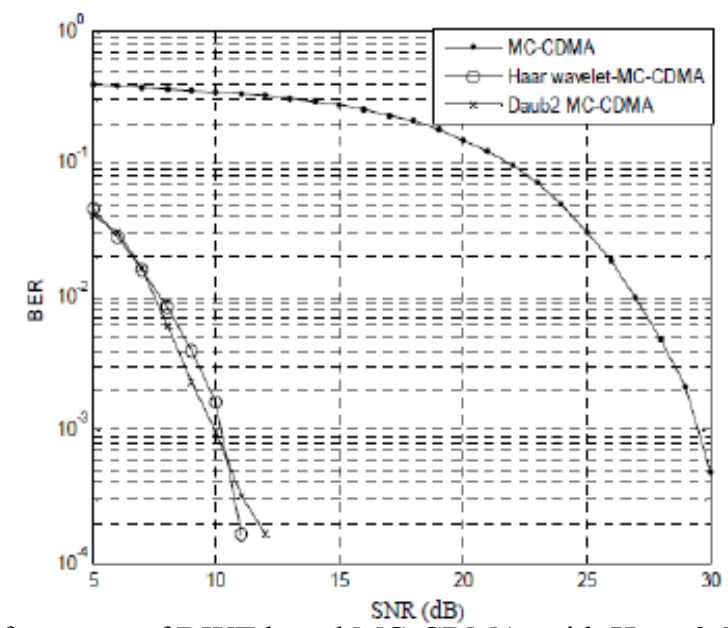

Fig. 6. BER Performance of DWT based MC-CDMA with Haar \& Daubechies wavelets

"Fig . 6" shows the BER performance of of FFT based MC-CDMA system and DWT based MC-CDMA system with Haar and Daubeches wavelet. Here we have taken 64- sub carrier and carrier frequency of $900 \mathrm{MHz}$, The symbol period is $65 \mu \mathrm{sec}$ and the sub carrier modulation was 16-QAM. From the Fig.6 ,the FFT based MCCDMA system has at approximately $\mathrm{SNR}=29 \mathrm{~dB}$ at $\mathrm{BER}=10^{-3}$, while DWT based MC-CDMA system with Haar wavelet has at approximately $\mathrm{SNR}=5 \mathrm{~dB}$ at $\mathrm{BER}=10^{-3}$ and with daubechies approximate $5 \mathrm{~dB}$, which means a gain of about $24 \mathrm{~dB}$ has been achieved. It is clear from Fig. 6 that DWT based MC-CDMA system better BER performance as compared to FFT based MC-CDMA system.

Fig. 6. BER Performance of DWT based MC-CDMA with Haar \& Daubechies wavelets

\section{CONCLUSIONS}

In this work we have shown that DWT based MC-CDMA system is more bandwidth efficient than FFT based MC-CDMA system .From the simulation result it is found that DWT based MC-CDMA system achieves better BER from $10^{-3}$ to $10^{-1}$ as compared to FFT based MC-CDMA system under AWGN channel and flat fading channel. DWT based MC-CDMA system achieves marked improvement with Haar wavelet over FFT based MC-CDMA system. The special feature of DWT is that it has high degree of sidelobe suppression and the loss of orthogonality leads to lesser inter-symbol interference (ISI) and inter-carrier interference(ICI) than FFT based system. DWT based multi-carrier systems has better spectral containment of channels since it does non use cyclic prefix. Also DWT based multi-carrier systems with diversity techniques and / or error correcting coding needs investigation in future for better performance.

\section{REFERENCES}

[1]. J. A. C. Bingham, "Multicarrier modulation for data transmission: an idea whose time has come,"IEEE Communications Magazine, vol. 28, no. 5, pp. 5-14, May 1990.

[2]. Shinsuke Hara, and Ramjrr Prasad. "Multicarrier Techniques for 4G Mobile Communications," Artech House, Boston. London, (2003).

[3]. J. G. Proakis, Digital communications, 3rd ed. Mc Graw Hill international editions, 1995

[4]. N. Erdol, F. Bao, and Z. Chen, "Wavelet modulation: a prototype for digital communication systems," in IEEE Southcon Conference, 1995 , pp. 168-171.

[5]. C. K. Chui, An introduction to wavelets. Academic Press, 1992, vol. 1.

[6]. R. Dilmirghani, M. Ghavami, “Wavelet Vs Fourier Based UWB Systems”, 18th IEEE International Symposium on Personal, Indoor and Mobile Radio Communications, pp.1-5, September 2007.

[7]. A. Akansu, P. Duhamel, X. Lin, and M. de Courville, “Orthogonal transmultiplexers in communications: a review,"IEEE Transactions on Signal Processing, vol. 46, no. 4,pp. 979-995, April 1998.

[8]. Y. Li, G.L. Stuber, Orthogonal Frequency Division Multiplexing for Wireless Communications, Springer, 2006.

[9]. T.S. Rappaport, Wireless communications: principles and practice.Vol. 2, 1996: Prentice Hall PTR

[10]. Andrea Goldsmith, Wireless Communications, Cambridge University Press, (2005).

[11]. Y. Zhang, S. Cheng, A novel multicarrier signal transmission system over multipath channel of low-voltage power line, IEEE Trans. Power Deliv. 19 (4) (October 2004) 1668-1672.

[12]. Deepak Gupta,Torry Harris,Vipin B Vats,Kamal K.Garg, "Performance Analysis of DFT-FDM,DCT-OFDM and DWT-OFDM Systems in AWGN Channel", The fourth International Conference on Wireless and Mobile Communications ICWMC 2008 IEEE Computer Society Washington,USA.

[13]. F. Dovis, M. Mondin, and F. Daneshgaran, "Performance of wavelet waveforms over linear and non-linear channels," in Proceedings of Wireless Communications and Networking Conference (WCNC), 1999, pp. 1148-1152.

[14]. K.P. Soman and K.I. Ramachandran, "Insight into Wavelets from Theory to Practice", PHI Publication 
[15]. Hiroki Harada, Marco Hernandez and Ryuji Kohno, Multiband and Multicarrier Wavelet PacketMultiplexing for UWB Transmissions, Proceedings of the 2008 IEEE International Conference on Ultrawideband (ICUWB2008), Vol. 3, 2008.

[16 ]. Mallat S. A wavelet tour of signal processing. second ed. California: Academic Press, Elsevier; 1999.

[17 ]. Rabindra Bhojray, and Subhendu Kumar Behera. "Multi-resolution Image Fusion using Marker Controlled Watershed Transform and DWT". International Journal of Electronic communication and Computer Engineering 3.5 (2012): page 1037-1040.volume 3,1ssue 5, sept 2012 .

[18]. Cotronei M. et al, “Multiwavelet Analysis and Signal Processing”, IEEE, Transaction on Circuitsand Systems II.volume45,issue 8,page 970-987,Aug1998.

[19]. A.Akansu, P.Duhamel, X.Lin, and M.de Courville, “Orthogonal Tran multiplexers in communication: a review," IEEE Transactions on signal processing, vol.46no.4, pp.979-995, April 1998.

[20]. Baoguo Yang; Letaief, K.B.; Cheng, R.S.; Zhigang Cao, Channel estimation for OFDM transmission in multipath fading channels based on parametric channel modeling, IEEE Transactions on Communications, Vol. 49, No. 3, 2001, pp. $467-479$.

[21]. Lee Y-S, Shin H-C,KimH-N. Channel estimation based on a time-domain threshold for OFDM ystems. IEEE Trans Broadcast 2009;55(September (3)):656-62.

[22]. Yuan-Pei Lin, See-May Phoong, “Perfect Discrete Multitone Modulation with Optimal Transceivers”, IEEE Transactions on Signal Processing, Vol. 48 no. 6, June 2000

[23 ]. W. Saad, N. El-Fishawy, S. EL-Rabaie, and M. Shokair, An Efficient Technique for OFDM System Using Discrete Wavelet Transform, Springer-Verlag Berlin Heidelberg, pp. 533-541, 2010.

[24]. R.M. Vitenberg, A wavelet based filtered multi-tone, in: IEEE 10th International Conference on Advanced Communication Technology, ICACT 2008, vol. 3, 17-20 February 2008, pp. 1849-1853.

[25]. Kaiser S. “On the Performance of Different Detection Techniques for MC-CDMA-CDMA in Fading Channels," IEEE ICC'95. pp. 2059- 2063, June (1995).

[26]. G. Bachman, L. Narici, and E. Beckenstein, Fourier and wavelet analysis. Springer, 2000.

[27]. G. W. Wornell, "Emerging applications of multirate signal processing and wavelets in digital communications, "in Proceedings of the IEEE, vol. 84, 1996, pp. 586-603.

[28]. L. Hanzo, W. Webb, and T. Keller, Single- and multicarrier quadrature amplitude modulation - Principles and applications for personnal communications, WLANs and broadcasting. John Wiley \&Sons, 2000

[29]. I. Barhumi et al, "Time-Varying FIR Equalization for Doubly-Selective Channels", IEEE Trans. On Wireless Comm., Vol. 4, no. 1, Jan. 2005 .

[30]. F. Dovis, M. Mondin, and F. Daneshgaran, "Performance of wavelet waveforms over linear and non- linear channels," in Proceedings of Wireless Communications and Networking Conference (WCNC), 1999, pp. 1148-1152. 